\title{
Learning from Anaesthetists: A Technique for Safe and Effective Facial Nerve Monitoring
}

\author{
Jayan George ${ }^{1, * \mathbb{C}}$, Anton Alatsatianos ${ }^{2}$, Amir Farboud $^{3}$ and Laysan Pope ${ }^{3}$ \\ 1 General Surgical Department, Sheffield Teaching Hospitals NHS Foundation Trust, Herries Road, \\ Sheffield S5 7AU, UK \\ 2 ENT Head and Neck surgery Department, Queen Elizabeth University Hospital, 1345 Govan Road, \\ Glasgow G51 4TF, UK; alatsatianos@doctors.org.uk \\ 3 ENT Head and Neck surgery Department, Morrison Hospital, Heol Maes Eglwys, Swansea SA6 6NL, UK; \\ amirfarboud@doctors.net.uk (A.F.); laysan.pope@wales.nhs.uk (L.P.) \\ * Correspondence: jayan.george@aol.com; Tel.: +44-1756-205-666
}

Received: 17 October 2018; Accepted: 6 March 2019; Published: 11 March 2019

check for updates

\begin{abstract}
To develop a technique to reliably secure facial nerve monitoring electrodes, Neurosign ${ }^{\mathrm{TM}}$ facial nerve monitor electrodes were attached as per the manufacturer's instructions. The electrodes were secured with $1 / 2$ inch steristrips ${ }^{\mathrm{TM}}$ before connecting leads were brought to the contralateral side of the face and a single torque loop was created and secured with either more Steri-Strips ${ }^{\mathrm{TM}}$, Micropore tape $^{\mathrm{TM}}$, or a Tegederm ${ }^{\mathrm{TM}}$. By creating a single torque loop, a buffer between the electrode and its anchor point to the contralateral face was formed. This allowed for the secure attachment of leads from the electrodes by removing tension, thereby reducing the likelihood of displacement. We have used this technique successfully for the last three years in over 50 parotid procedures and 50 middle ear explorations. Facial nerve monitoring is an important surgical tool used in otology and head and neck surgery. Using torque loops when preparing a patient for surgery prevents the dislodgement of electrodes during operation, thereby ensuring the safety of the patient in a sometimes perilous environment.
\end{abstract}

Keywords: otology; parotid gland; facial nerve

\section{Introduction}

Post-operative facial nerve paralysis is a devastating complication of otological and head and neck surgery. Sequela includes cosmetic disfigurement and ophthalmological complications causing significant morbidity to a patient. The current literature estimates that up to $7 \%$ of patients will suffer permanent facial nerve injury following parotid surgery [1]. Facial nerve monitoring is a common adjuvant used during procedures that can place the facial nerve at risk of injury, although its routine use in parotid surgery remains controversial [2]. In the UK, over $80 \%$ of surgeons routinely use it [2].

A prospective questionnaire in the United States reported that facial nerve monitors had been used in $60 \%$ of parotid surgery performed by ear, nose and throat (ENT) surgeons [1]. The authors have also reported that respondents to their survey were $20.8 \%$ less likely to have a lawsuit from parotid gland surgery if they employed facial nerve monitoring in their practice [1]. Malpractice lawsuits involving the salivary glands is one of the most expensive indemnities against otolaryngologists in the United States, the majority of which are settled out of court [3]. A retrospective study of US civil trials from 1987-2011 revealed 26 cases of malpractice for parotid surgery, 10 of which involved injury to the facial nerve, with the largest indemnity awarded of $\$ 7,272,087$ [3].

Although the standard of care in parotid surgery has yet to be established, surgeons who opt to use facial nerve monitoring must ensure that the electrodes and leads are secured and tested. Common 
to many otolaryngology operations, the intra-operative area around a patient's head and neck is crowded by anaesthetic equipment including, but not limited to, a bispectral index monitor ${ }^{\mathrm{TM}}$ and endotracheal tube. Consequently, facial nerve monitoring leads that are loosely attached are at risk, and can easily become dislodged, thus compromising safety. To reduce this risk, we have derived a technique, borrowed from our anaesthetic colleagues, that we use routinely in all of our parotid gland and middle ear surgeries to manage and secure facial nerve monitoring equipment.

\section{Material and Methods (Technical Description)}

Neurosign ${ }^{\mathrm{TM}}$ facial nerve monitor electrodes were attached as per manufacturer's instructions [4]. Electrodes were secured with $1 / 2$ inch Steri-Strips ${ }^{\mathrm{TM}}$. Connecting leads were then brought to the contralateral side of the face and a single torque loop was created and secured with either more Steristips ${ }^{\mathrm{TM}}$, Micropore tape $\mathrm{T}^{\mathrm{TM}}$, or a Tegederm ${ }^{\mathrm{TM}}$ (Figures 1 and 2). By using a single torque loop, there is a gap between the electrode and its anchor point to the contralateral face, therefore there is little or no tension on the relevant electrode, making it much less likely to become dislodged during surgery.

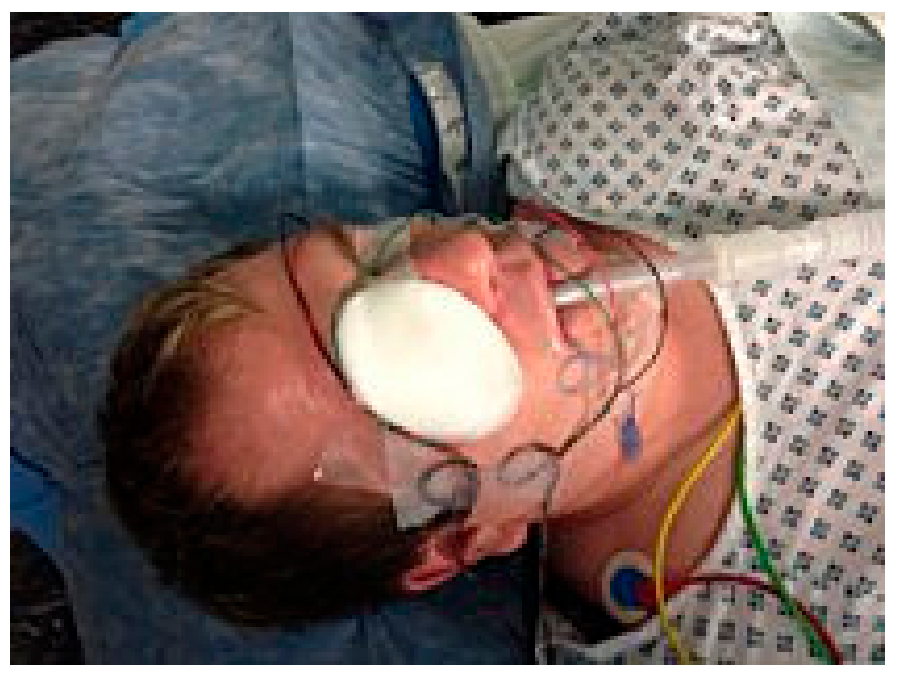

Figure 1. Electrodes held in place with Elastoplast ${ }^{\mathrm{TM}}$ Wires from the electrodes were brought to the contralateral side of the face.

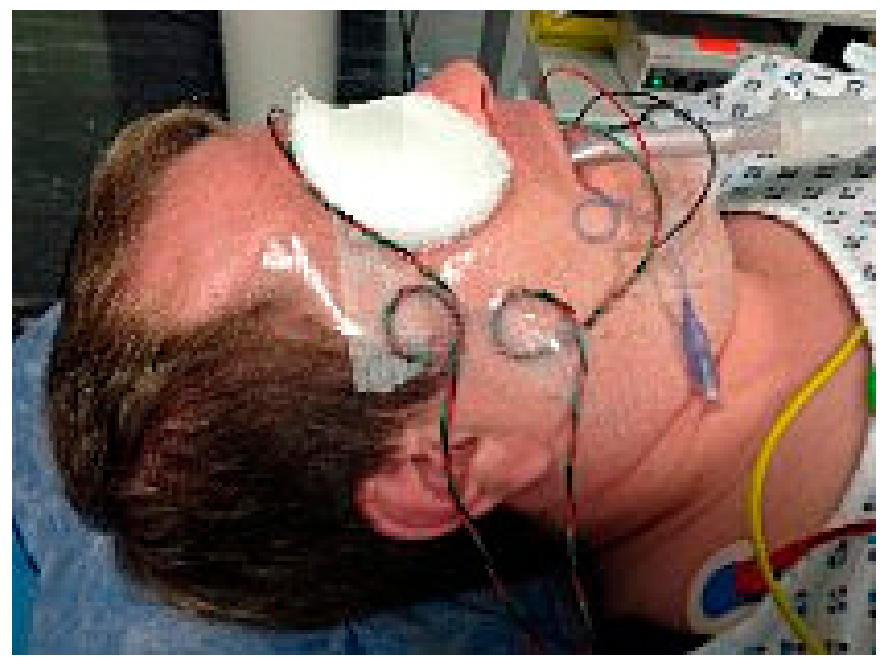

Figure 2. Wires were secured with Elastoplast ${ }^{\mathrm{TM}}$ in torque loops. 


\section{Results}

Our described technique allows for the safe and secure attachment of nerve electrodes, thereby reducing their accidental displacement. We have deployed this technique successfully for the last three years in parotid and middle ear surgery. In the combined caseload of the senior authors including over 50 parotid and 50 middle ear cases over three years, there were no dislocations of the electrodes, and no permanent facial nerve palsies.

\section{Discussion}

Facial nerve injury is a dreaded complication of otological and head and neck surgery. The use of nerve monitoring has become routine practice for many clinicians operating on the parotid gland and is becoming the standard of care in middle ear surgery. It can help reduce surgical complications and any subsequent litigation, although its true efficacy has yet to be ascertained [5]. Displacement of the electrodes whilst operating can compromise the safety offered by nerve monitoring devices. The described technique of using torque loops is routine in our practice, thereby reducing electrode displacement and adding a further level of security.

\section{Conclusions}

Facial nerve monitoring is an important surgical tool used in otology and head and neck surgery. Complications from facial nerve injuries can cause significant morbidity to patients and litigation against clinicians. Facial nerve monitoring is only effective if the electrodes are securely attached to the patient. Using torque loops when preparing a patient for surgery prevents the dislodgement of electrodes whilst operating and has become our adopted practice, which we feel has raised the standard of care we offer our patients.

Author Contributions: J.G.: Preparing the manuscript, editing, and proofing; A.A.: Developing and proofing the manuscript; A.F.: Editing, original idea, innovator, and proofing; L.P.: Editing, concept development, and proofing.

Funding: This research received no external funding.

Conflicts of Interest: The authors declare no conflict of interest.

\section{References}

1. Lowry, T.R.; Gal, T.J.; Brennan, J.A. Patterns of use of facial nerve monitoring during parotid gland surgery. Otolaryngol. Neck Surg. Off J. Am. Acad. Otolaryngol. Neck Surg. 2005, 133, 313-318. [CrossRef] [PubMed]

2. Hopkins, C.; Khemani, S.; Terry, R.M.; Golding-Wood, D. How we do it: Nerve monitoring in ENT surgery: Current UK practice. Clin. Otolaryngol. 2005, 30, 195-198. [CrossRef] [PubMed]

3. Hong, S.S.; Yheulon, C.G.; Sniezek, J.C. Salivary gland surgery and medical malpractice. Otolaryngol. Head Neck Surg. 2013, 148, 589-594. [CrossRef] [PubMed]

4. Neurosign. Neurosign 400 Operating Manual. 2011. Available online: http://www.neurosignsurgical.com/ wp-content/uploads/2012/08/Neurosign-400-Operating-Manual.pdf (accessed on 28 March 2017).

5. Mahmood, K.; Williams, G.S.; Morgan, N. Postparotidectomy facial nerve paralysis: Peripheral versus proximal identification. $B$-ENT 2010, 6, 117-121. [PubMed] 